\title{
Experiencia del grupo de cirugía bariátrica y metabólica del Hospital Universitario de Neiva durante un año
}

\author{
Study of the metabolic and bariatric surgery group in the university hospital \\ of Neiva over a year
}

\begin{abstract}
Angie J. Barreto Sánchez', Mayra A. Carballo Bernal', Diego Fernando Chimbaco Bonilla', Adriana L. León Ortiz', Javier Leonardo Mancini Castrillón', Guillermo Pinzón Santos', Lithey Cristina Poveda Conde, Arnold Salcedo Cordoba', Héctor Adolfo Polanía ${ }^{2}$
\end{abstract}

\begin{abstract}
Resumen
Objetivo. Describir los resultados quirúrgicos, metabólicos y de percepción corporal obtenidos en pacientes sometidos a Cirugía Bariátrica, para establecer la efectividad del procedimiento en pacientes obesos. Materiales y métodos: estudio serie de casos. Se revisaron 35 historias clínicas de pacientes intervenidos quirúrgicamente por el equipo de Cirugía Bariátrica entre Septiembre del 2011 a Septiembre del 2012. Resultados: El $91 \%$ de los pacientes presentó una o más comorbilidades asociadas, siendo las gastrointestinales las más frecuentes. La técnica más aplicada fue el Bypass gástrico. El $12 \%$ presentó complicaciones posquirúrgicas. Todos redujeron su peso con una media de pérdida de índice de masa corporal (IMC) al primer y sexto mes de 5.4 y $11,6 \mathrm{~kg} / \mathrm{m} 2$ respectivamente. Los pacientes con asa alimentaria a $150 \mathrm{~cm}$ tuvieron una mayor pérdida peso al sexto mes $(2 \mathrm{~kg} / \mathrm{m} 2$ más) respecto a aquellos con asa alimentaria de $100 \mathrm{~cm}$. El $98 \%$ reportó disminución de los síntomas gastrointestinales y el $30 \%$ redujo las cifras de glicemia. El $20 \%$ de pacientes diabéticos y el $12,5 \%$ de los hipertensos continuaron con medicamentos para su enfermedad. Conclusiones. La Cirugía Bariátrica es una técnica efectiva para reducir el IMC y mejorar las comorbilidades asociadas a la obesidad. Así mismo, disminuye la necesidad del uso de medicamentos. La autopercepción de la imagen corporal posquirúrgica mejora ostensiblemente.
\end{abstract}

Palabras clave: cirugía bariátrica, bypass gástrico, obesidad, índice de masa corporal, imagen corporal.

\begin{abstract}
Objective: Describe the surgical and metabolic results and changes to body image in patients undergoing bariatric surgery, in order to establish the effectiveness of the procedure in obese patients. Method and materials: Case study. The study examined the medical history of 35 patients who underwent bariatric surgery between September 2011 and September 2012. Results: 91\% of patients had one or more associated comorbidities, of which gastrointestinal disorders were the most common. The most common technique was the gastric bypass. $12 \%$ developed postoperative complications. All patients reduced their bodyweight with an average body mass index (BMI) loss of 5.4 and $11.6 \mathrm{~kg} / \mathrm{m} 2$ in the first and the sixth month respectively. Patients with a small intestine of $150 \mathrm{~cm}$ lost more weight by the sixth month $(2 \mathrm{~kg} / \mathrm{m} 2$ more) than those with a small intestine of $100 \mathrm{~cm} .98 \%$ reported a decrease in gastrointestinal symptoms and glycaemic levels reduced in $30 \% .20 \%$ of diabetic patients and $12.5 \%$ of hypertensive patients continued
\end{abstract}

1. Estudiante Programa de Medicina, Universidad Surcolombiana, Neiva. Colombia.

2. Docente Programa de Medicina, Facultad de Salud, Universidad Surcolombiana, Colombia. Hospital Universitario de Neiva.

Correspondencia: Héctor Adolfo Polanía. Correo electronico: hectoradolfopolania@yahoo.es.

Recibido: 19/08/2013-Revisado: 30/10/2014-Aceptado: 01/11/2015 
taking medication for their conditions. Conclusions: Bariatric surgery is an effective technique to reduce BMI and improve comorbidities associated with obesity, thereby reducing the necessity of the use of medication. Self-perception of post-surgical body image improves considerably.

Key words: bariatric surgery, gastric bypass, obesity, body mass index, body image.

\section{Introducción}

El sobrepeso y la obesidad, han sido establecidos por La Organización Mundial de la Salud, como el quinto factor de riesgo de muerte en el mundo. Se calcula 2.8 millones de fallecimientos de adultos al año como causa de estas, además de la alta morbilidad asociada como la diabetes (44\%), cardiopatías isquémicas $(23 \%)$ y algunos tipos de cáncer $(41 \%)^{1}$. En Colombia se ha establecido una cifra de 12 millones de personas con sobrepeso u obesidad, cerca de 1 millón de personas con diabetes y 2 millones de individuos con hiperglucemia en ayunas o intolerancia a los carbohidratos, entidades que preceden a la diabetes mellitus tipo $2^{2}$.

Según la Encuesta Nacional de la Situación Nutricional (ENSIN 2010) ${ }^{3}$ en Colombia dirigida por el ICBF, realizada del 2008 al 2010 en 50.670 hogares, en la población entre los 5 y 17 años muestra que 1 de cada 6 niños y adolescentes presenta sobrepeso u obesidad; esta relación aumenta a medida que se incrementa el nivel del SISBEN y el nivel educativo de la madre, igualmente es mayor en quienes viven en las zonas urbanas. La prevalencia de sobrepeso u obesidad ha aumentado un $25,9 \%$ en el último quinquenio; entre los adultos de 18 a 64 años, el $62 \%$ de las mujeres y $39.8 \%$ de los hombres tienen obesidad abdominal, el cual representa un importante factor de riesgo para enfermedades cardiovasculares. Todo esto evidencia que el exceso de peso en la población adolescente y adulta tiene una tendencia al incremento, a pesar de los esfuerzos para su prevención.

Los tratamientos médicos tradicionales no siempre dan los resultados deseables para combatir la cantidad de efectos y perjuicios que pueden acontecer a una persona obesa, ya sea por ineficacia de los medicamentos, por falta de poder adquisitivo por parte de los pacientes o por falta de adherencia a los esquemas terapéuticos. Por ejemplo, en diabetes mellitus a pesar de los avances en farmacoterapia, menos del 50\% de los pacientes alcanza y mantiene los niveles terapéuticos de control de la glicemia ${ }^{4}$. Por lo tanto, la Cirugía Bariátrica se perfila como una estrategia moderna, no sólo para la reducción de peso, sino también como un método de control de cifras de glicemia y de colesterol en sangre, por lo cual también se conoce como Cirugía Metabólica. Desde el 2011, la Federación Internacional de Diabetes la reconoció como una alternativa terapéutica ${ }^{5}$. Otras ventajas, incluyen la reducción de factores de riesgo cardiovasculares, mejoría drástica de la apnea del sueño, el reflujo, la fatiga, además del beneficio de una apariencia física más agradable para el paciente, con la consiguiente mejora de su autoestima y desempeño laboral.

Los procedimientos que se practican con mayor frecuencia para facilitar la pérdida de peso incluyen, entre otros: bypass gástrico en Y de Roux, manga gástrica, banda gástrica ajustable, banda gástrica no ajustable, derivación biliopancreática y gastroplastia vertical con bandas ${ }^{6}$; actualmente, la que menos se realiza es ésta última ${ }^{7}$. A pesar de que se ha demostrado estas intervenciones como efectivas en la reducción de peso, pueden presentarse eventos adversos como una pérdida insuficiente de peso, ganancia de peso u otra complicación; y están muy relacionadas con el tipo de intervención quirúrgica seleccionada inicialmente para el manejo de la obesidad mórbida ${ }^{8-11}$.

Este estudio presenta información sobre la evolución de pacientes que fueron sometidos a este procedimiento quirúrgico metabólico, realizado por el grupo de Cirugía bariátrica del Hospital Universitario de Neiva por un periodo de 1 año, con el fin de generar aportes con respecto a la epidemiología local, y evaluar la efectividad de la cirugía frente a otros abordajes terapéuticos.

\section{Materiales y métodos}

Se realizó un estudio retrospectivo, descriptivo observacional, tipo serie de casos en el cual se realizó una revisión manual de 35 historias clínicas, de las cuales se incluyeron 33 , ya que en el periodo de recolección de datos posterior a la cirugía no fue posible localizar a dos pacientes con la información personal suministrada. Se obtuvo los datos del seguimiento postquirúrgico de cada paciente en el sistema SIIGHO de consulta externa. A cada paciente, previo consentimiento informado se le realizó una entrevista para evaluar la imagen corporal a través de la escala de Stunkard y Stellard (1990), modificada por Collins (1991), un instrumento que involucra la estimación del tamaño (dimensión perceptual); el ejercicio consiste en que el sujeto debe identificarse con una de la figuras de la escala que se encuentra entre 1-9, determinando la satisfacción oculo-espacial que tiene de sí mismo ${ }^{11}$. Se realizaron dos mediciones: una única medición pre-quirúrgica y otra post quirúrgica únicamente al momento de la realización del estudio.

Las condiciones médicas que se tuvieron en cuenta para observar los cambios secundarios a la cirugía bariátrica fueron: Tensión arterial, Frecuencia Cardiaca, Peso, circunferencia abdominal, Glicemia, HbA1, medicamentos y comorbilidades. Además, se describió el tiempo operatorio, los días de hospitalización, las complicaciones, la mortalidad y la morbilidad del procedimiento quirúrgico.

El análisis de datos fue realizado mediante el programa estadístico Epi Info v.3.4.3 desarrollado por el CDC año 2007 y Microsoft Office Excel 14.0.0 por Microsoft corporation en el año 2013. El tipo de análisis fue de tipo descriptivo con apoyo en gráficas, se utilizó medidas de tendencia central para describir las características sociodemográficas, el esta- 


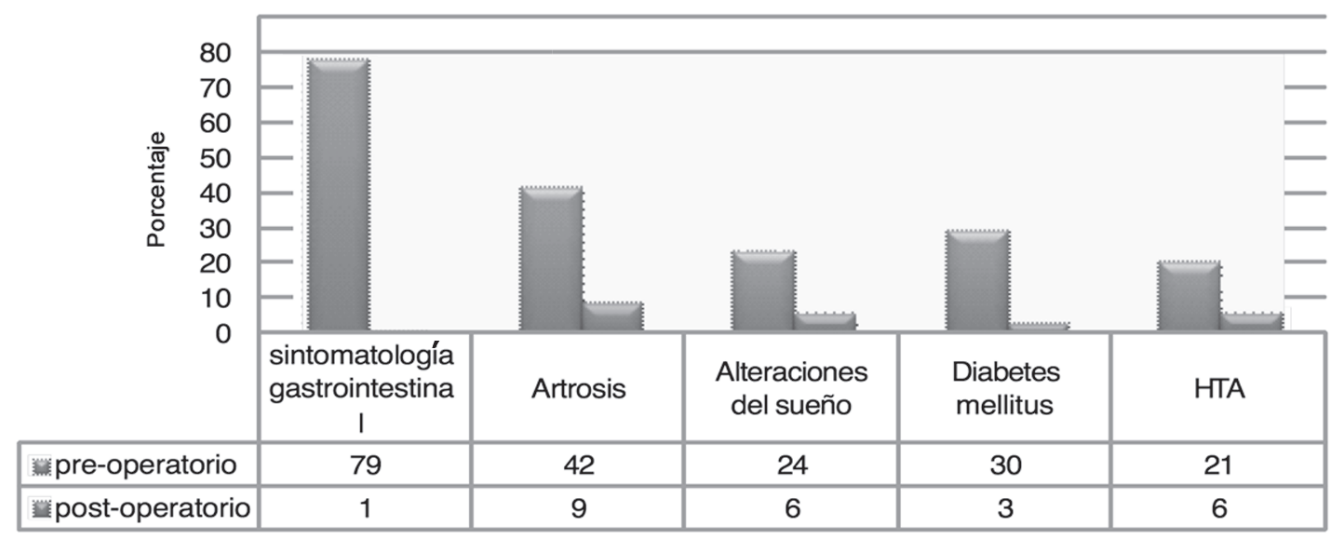

Figura 1. Relación estadística de las comorbilidades pre-operatorias y post-operatoria.

do pre-quirúrgico y actual de los pacientes, la técnica quirúrgica más utilizada y las complicaciones que se presentaron en los pacientes. Para analizar la pérdida de peso post quirúrgico en relación con la longitud del asa alimentaria, así como con la mejoría en las cifras de glicemia en pacientes diabéticos, se empleó el coeficiente de correlación entre dos variables numéricas. Debido al tamaño de la muestra el propósito es caracterizar el grupo poblacional intervenido sin pretensiones de extrapolar los datos a otros grupos de individuos no incluidos en el estudio.

\section{Resultados}

En total se intervinieron quirúrgicamente 35 pacientes entre septiembre del 2011 a septiembre del 2012, de los cuales se incluyeron en nuestra investigación 33 ya que a 2 de ellos no se logró hacerle el seguimiento respectivo.

De los 33 pacientes el 88\% (29) eran mujeres. El rango de edad de los pacientes intervenidos está entre 20 a 54 años, con una media de 36 años. Dado que la mayoría de las mujeres intervenidas eran amas de casa, el hogar representa el $62 \%$ de las ocupaciones.

El $78.8 \%$ de las personas procedían del municipio de Neiva, siendo los estratos socioeconómicos bajos (1 y 2) el $90 \%$. El régimen de salud al que más pertenecían era el subsidiado con el 73\%. El 15,62\% de los pacientes presentaba algún antecedente toxicológico, el $6.25 \%$ de tabaquismo y 9.2\% de alcoholismo.

El promedio de tiempo de hospitalización de los pacientes intervenidos fue de 2 días y el $12 \%$ de ellos presentó alguna complicación postquirúrgica (Tabla1).

El 91\% de nuestros pacientes presentaba algún tipo de comorbilidad como alteraciones gastrointestinales, artrosis, síndrome metabólico entre otros, a lo largo del seguimiento, se encontró que la sintomatología gastrointestinal disminuyó en un 78\% (Figura 1). Las alteraciones Gastrointestinales pre quirúrgicas más frecuentes fueron gastritis, esteatosis Hepática y colelitiasis (Figura 2).

Las cifras de glicemia disminuyeron en el 30\% de los pacientes en los primeros 6 meses, un paciente permaneció
Tabla 1. Tipo de complicaciones presentadas en los pacientes intervenidos quirúrgicamente.

\begin{tabular}{lcc}
\hline Complicaciones & $\begin{array}{c}\text { Número } \\
\text { de pacientes }\end{array}$ & $\begin{array}{c}\text { Porcentaje de } \\
\text { de pacientes }\end{array}$ \\
\hline Desórdenes & 1 & $3 \%$ \\
hidro-electrolíticos & 1 & $3 \%$ \\
ISO & 1 & $3 \%$ \\
Embarazo & & \\
\hline
\end{tabular}

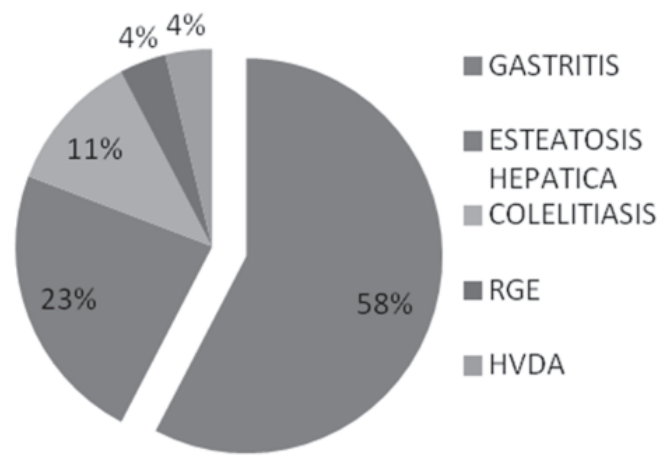

Figura 2. Alteraciones gastrointestinales pre-quirúrgicas.

controlado sin utilizar medicamentos (Figura 3) y el $80 \%$ presentó menor consumo de estos para controlar las cifras de glicemia (Tabla 2). De igual manera los pacientes previamente hipertensos presentaron una evolución favorable en sus cifras tensionales. El 57\% de ellos requieren actualmente una menor dosis o un menor número de antihipertensivos para mantener cifras tensionales normales. (Tabla 2).

Todos los pacientes presentaron reducción de peso, representado en la disminución del IMC. El promedio de pérdida en $\mathrm{Kg} / \mathrm{m}^{2}$, al mes de haber realizado el procedimiento quirúrgico fue de $5,4 \mathrm{Kg} / \mathrm{m}^{2}$, y fue mayor $\left(5,9 \mathrm{~kg} / \mathrm{m}^{2}\right)$ en aquellos pacientes con un IMC $>$ a $50 \mathrm{Kg} / \mathrm{m}^{2}(21,4 \%$ ) (Figura 4). A los seis meses de control hubo una diminución mucho más significativa del IMC en todos los pacientes con un promedio de 


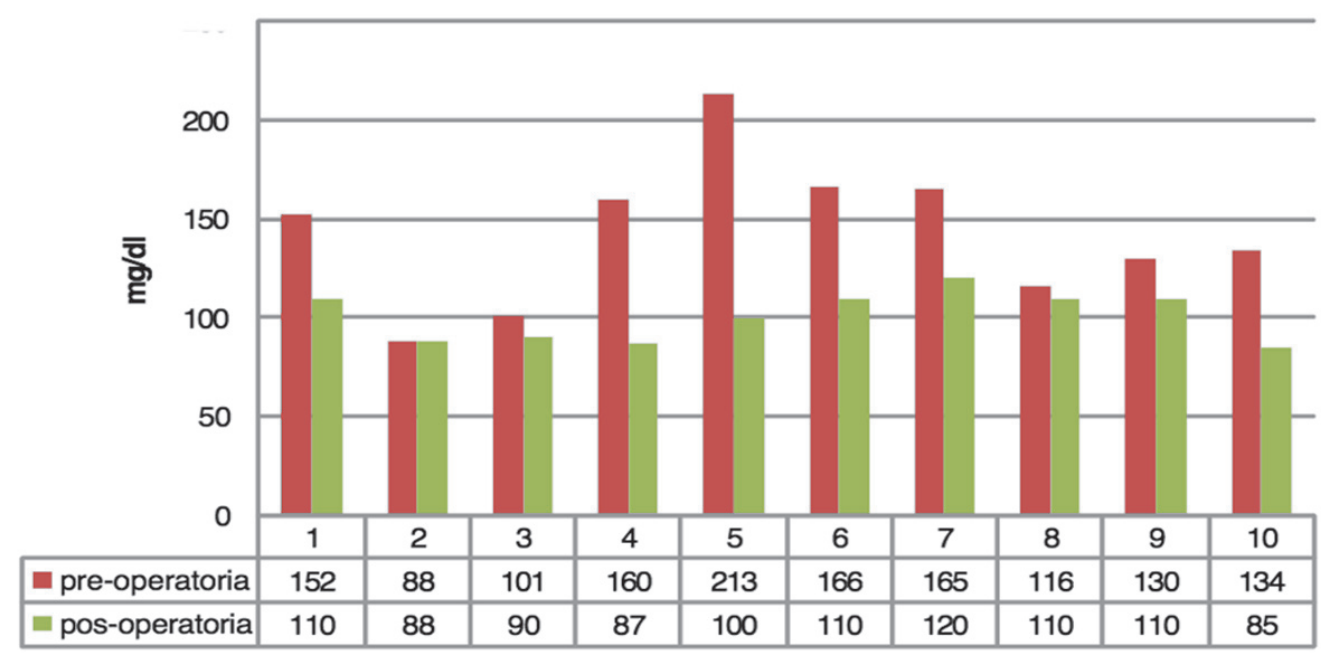

Figura 3. Seguimiento de la glicemia pre-operatoria y post-operatoria en los pacientes con Diabetes Mellitus tipo II.

Tabla 2. Pacientes con diagnóstico previo de diabetes mellitus tipo 2 e hipertensión arterial que actualmente consumen medicamentos.

\begin{tabular}{lll}
\hline Diagnóstico & Sí & No \\
\hline Diabetes mellitus tipo II & 2 & 8 \\
Hipertensión arterial & 4 & 4 \\
\hline
\end{tabular}

Dre $0 x$

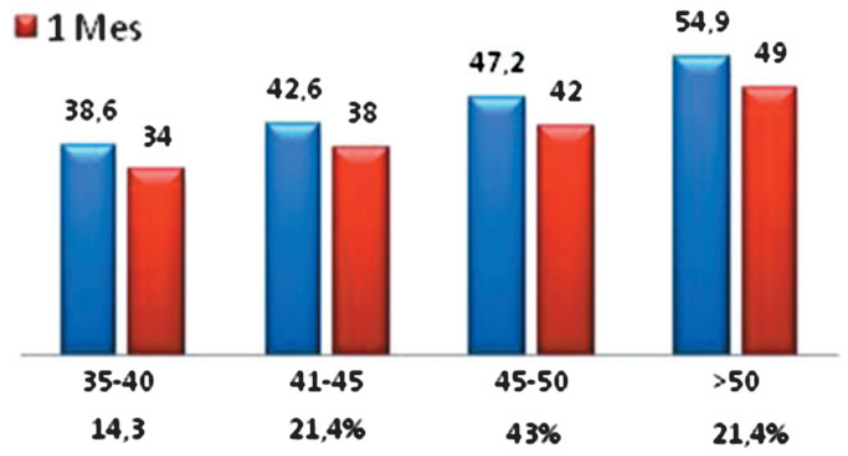

Figura 4. Evolución del índice de masa corporal (IMC) 1 mes.

pérdida acumulada de $11,6 \mathrm{~kg} / \mathrm{m}^{2}$, siendo más importante en los pacientes con un IMC $>50 \mathrm{Kg} / \mathrm{m}^{2}$ (Figura 5). Previo a la cirugía la media del índice de masa corporal (IMC) fue de 46.6 $\mathrm{kg} / \mathrm{m}^{2}$ y posterior a la cirugía se redujo considerablemente. (Figura 6).

A 32 de los pacientes se les practicó bypass gástrico y a uno (1) manga gástrica. La distancia del asa alimentaria a los

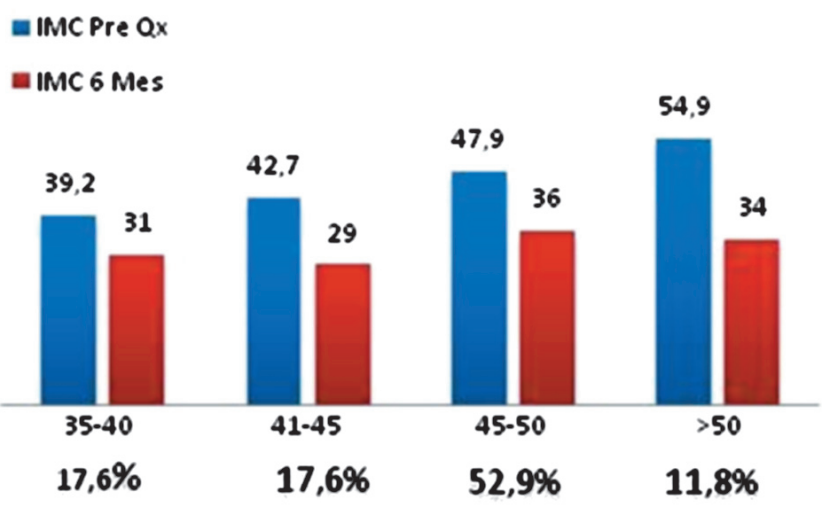

Figura 5. Evolución del índice de masa corporal (IMC) en los pacientes intervenidos quirúrgicamente durante 6 meses de seguimiento.

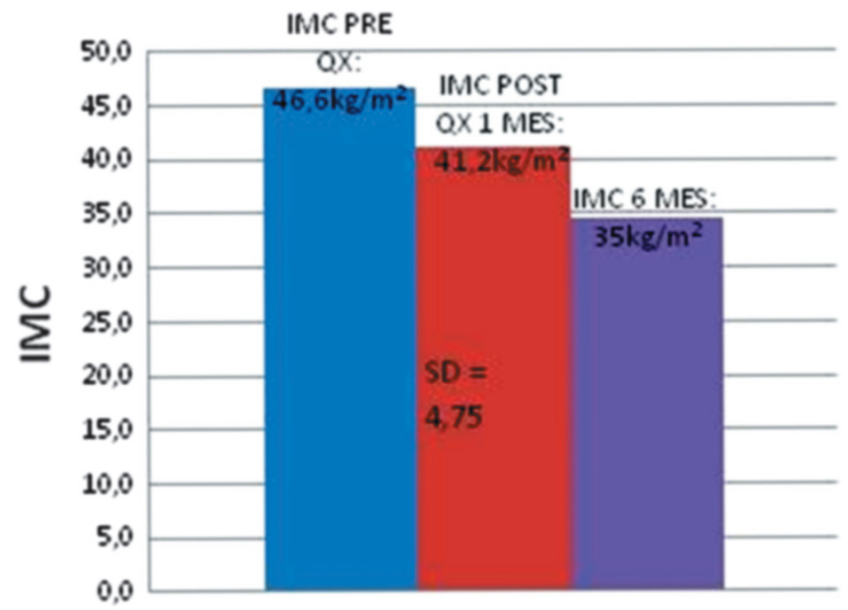

Figura 6. Relación del IMC pre-quirúrgico y a los 6 meses. 
pacientes con bypass varió según el IMC, fue de $100 \mathrm{~cm}$ para pacientes con IMC $<45 \mathrm{~kg} / \mathrm{m}^{2}(64 \%)$ y de $150 \mathrm{~cm}$, para IMC $>45 \mathrm{~kg} / \mathrm{m}^{2}$ (68\%). El IMC de los pacientes con un asa de 150 $\mathrm{cm}$ en comparación con los de asa de $100 \mathrm{~cm}$, disminuyó en el primer mes $1 \mathrm{Kg} / \mathrm{m}^{2}$, y $2 \mathrm{Kg} / \mathrm{m}^{2}$ más en el seguimiento a 6 meses (Figura 7). Lo anterior mostró una correlación positiva entre la longitud del asa y la disminución de peso entre los dos grupos comprobada mediante el coeficiente de correlación de Pearson con un resultado $=1$ favorable para el asa larga.

La percepción de la imagen corporal se calculó con base al método de Stukard y Stallard modificado por Collins, se realizó dos mediciones, una pre quirúrgica y una medición posquirúrgica al momento del estudio en donde se encontró que la mayoría de las pacientes $(85 \%)$ se ubicaban en la imagen nueve previo al procedimiento quirúrgico, y en la imagen cinco posterior a este respectivamente, manifestando satisfacción frente a su estado actual (Figura 8).

\section{Discusión}

La prevalencia de la obesidad es mayor en el sexo femeni$\mathrm{no}^{3,12}$, esto se refleja en que el mayor número de pacientes intervenidos quirúrgicamente fueron mujeres (88\%). Habitualmente los factores socioeconómicos como los ingresos, el nivel educativo y el acceso a la asistencia sanitaria han demostrado ser barreras importantes para recibir tanto atención médica básica como intervenciones médicas y quirúrgicas avanzadas $^{13}$, sin embargo en este estudio el $90 \%$ de los pa- cientes pertenecían a estratos socioeconómicos bajos y el $73 \%$ pertenecía al régimen de salud subsidiado.

Aunque, la literatura mundial ha establecido como indicación quirúrgica para cirugía bariátrica una edad entre 18-65 años, recientemente se ha encontrado que la relación riesgobeneficio también es favorable en pacientes mayores ${ }^{14-16}$. Ninguno de los pacientes presentó una edad superior a 65 años, la mayoría se encontraba en un intervalo de 30-34 años y la edad media fue de 36 años, resultados que se asemejan a 3 estudios de series de caso donde reportaron edades entre

\section{Asa $150 \mathrm{~cm}$ asa $100 \mathrm{~cm}$}

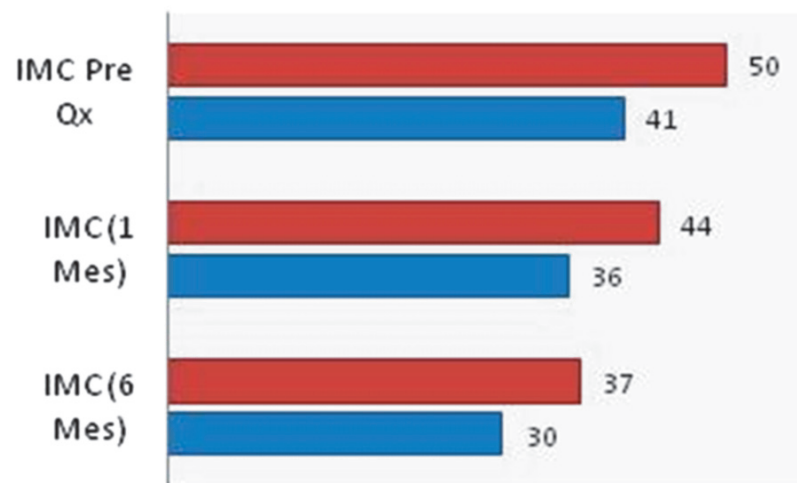

Figura 7. Correlación entre la longitud del asa alimentaria y el promedio de disminución de IMC entre el 1 y 6 mes posterior a la cirugía bariátrica.
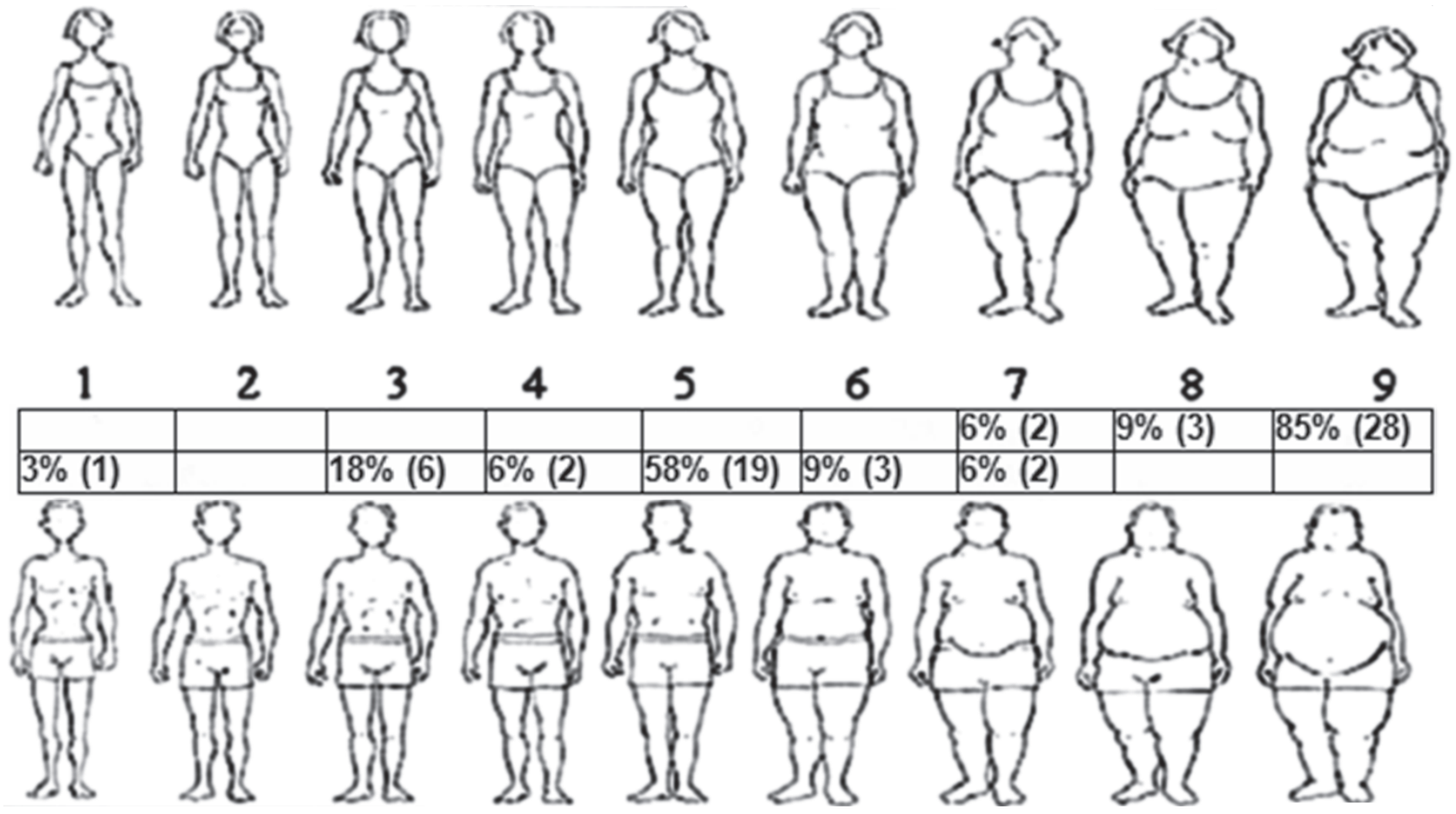

Figura 8. Seguimiento de la percepción corporal pre quirúrgico y post quirúrgico de los pacientes según el método de Stunkard y Stllard modificado por Collins. 
R.F.S Revista Facultad de Salud

Julio-Diciembre de 2015;7(2):15-22
Experiencia del grupo de cirugía bariátrica y metabólica del Hospital Universitario de Neiva durante un año los 37, 40 y 43 años en promedio, que han considerado una edad menor de 50 años como un factor predictor de buenos resultados ${ }^{17-19}$.

La obesidad se asocia con riesgo relativo de sufrir múltiples comorbilidades como diabetes, hipertensión arterial (HTA), hipercolesterolemia, asma, artritis, cáncer ${ }^{20}$. En esta investigación, se encontró que el 91\% de los pacientes presentaba algún tipo de enfermedad concomitante como alteraciones gastrointestinales $(52 \%)$, artrosis $(42 \%)$, Síndrome metabólico $(33 \%)$, alteraciones del sueño $(24 \%)$, Diabetes mellitus (30\%), Dislipidemia (21\%), HTA (21\%), Colelitiasis (9\%) entre otros. Las alteraciones gastrointestinales que se han descrito con mayor asociación con obesidad incluyen reflujo gastroesofágico, colelitiasis, hígado graso ${ }^{21-23}$; en este estudio las más frecuentes fueron gastritis crónica $(58 \%)$, esteatosis hepática (23\%), Colelitiasis (4\%), Reflujo Gastroesofágico (4\%). Entre los antecedentes, el 15,62\% de los pacientes presentaba algún antecedente toxicológico, actualmente el $12.62 \%$ de estos no presenta ningún consumo de sustancias.

El bypass gástrico ha demostrado tener los mejores resultados en cuanto a reducción de peso y mejoría de enfermedades asociadas a la obesidad, con un rango de seguridad bastante aceptable $24-26$. Al 97\% (32) de los pacientes se les practicó bypass gástrico. La diabetes constituye un factor de riesgo importante para las complicaciones postquirúrgicas, debido a que se afecta la cicatrización ${ }^{27}$. Las infecciones de herida quirúrgica luego de un "bypass" gástrico se reportan en 1-3\%. La mayoría son leves y rara vez se requiere de internamiento y antibióticos parenterales para su manejo ${ }^{28}$. También puede presentarse déficit de absorción de hierro, vitamina B12 y deficiencias de vitaminas liposolubles, así como también, anormalidades electrolíticas $^{29}$. En este estudio se encontró que el 3\% de los pacientes presentó Infecciones del Sitio Operatorio (ISO) y el 3\% desórdenes hidroelectrolíticos.

La pérdida de peso es bastante acelerada en los 3 primeros meses postoperatorios y luego se estabiliza. Todos los pacientes presentaron un descenso del IMC, la mayor pérdida de peso se obtuvo durante el primer mes. También se encontró una asociación entre la distancia del Asa alimentaria y la reducción del peso, la cual fue mayor en pacientes con un asa intestinal de $150 \mathrm{~cm}$. En algunos estudios, ni la reducción del diámetro de la anastomosis gastroyeyunal ${ }^{30,31}$ ni el largo del asa alimentaria han demostrado influir en la cantidad de peso reducido ${ }^{32}$. Por otra parte, un estudio en pacientes superobesos reportó mayor pérdida de peso en aquellos con un asa alimentaria de $150 \mathrm{~cm}$ que en pacientes con un asa de $70 \mathrm{~cm}$. Estos resultados, sin embargo, no se han reproducido en pacientes con obesidad de menor $\mathrm{IMC}^{33}$.

El éxito en cirugía bariátrica se define como la pérdida mínima del $50 \%$ del exceso de peso inicial del paciente obeso mórbido o una reducción del IMC a $35 \mathrm{~kg} / \mathrm{m} 2$ o menos. ${ }^{6}$, 21 Entre $25-30 \%$ de los pacientes sometidos a cirugía bariátrica pueden no llegar a perder suficiente peso ${ }^{34}$. Los mayores promedios de pérdida del exceso de peso que se logran a largo plazo son: $75 \%$ con la derivación biliopancreática, $60 \%$ con el bypass gástrico en $\mathrm{Y}$ de Roux, $50 \%$ con la manga gástrica y $23 \%$ con la banda gástrica $8,35,36$. En nuestro estudio el procedimiento fue efectivo en $34,92 \%$ de los pacientes, ya que todos alcanzaron un IMC menor a $35 \mathrm{~kg} / \mathrm{m}^{2}$. Además se encontró que los pacientes presentaron una disminución de los medicamentos para el control de la hipertensión y la glicemia, resultados similares a los del estudio sobre cirugía bariátrica contra terapia medica intensiva en obesos, publicado por Schauer et al, que encontraron una reducción importante del número de medicamentos necesarios para control de los síntomas de hiperglicemia por diabetes en los pacientes sometidos a la intervención ${ }^{4,37,38}$, mientras que en otros la diabetes se ha resuelto completamente ${ }^{17,39,40}$. Así mismo, se ha observado una reducción del riesgo cardiovascular, disminución sostenida de peso y mejor respuesta terapéutica en diabetes previa ${ }^{41}$, 42. También hubo mejoría de otras comorbilidades como artrosis, y alteraciones gastrointestinales. Este estudio evidencia y respalda otros estudios en los cuales la cirugía bariátrica no busca sólo conseguir una reducción del peso sino obtener mejoría de los trastornos metabólicos y de las enfermedades concomitantes asociadas a la obesidad ${ }^{4,43,44}$. No obstante, también influye en la autoestima de los pacientes, ya que todos en la escala Stukard y Stallard modificado por Collins presentaron una mejoría posquirúrgica de la percepción corporal.

Los resultados favorables de la cirugía bariátrica como una tasa menor de complicaciones, tiempo operatorio, tiempo de hospitalización y mortalidad, también dependen de la institución médica dónde se realice, la cual debe tener una acreditación de excelencia para estas intervenciones ${ }^{45}$. Aunque la conformación del equipo interdisciplinario de cirugía bariátrica del Hospital Universitario de Neiva es reciente, el curso de evolución de los pacientes intervenidos comparado con otros estudios es muy alentador.

\section{Referencias}

1. Organización Mundial de la Salud (OMS). Obesidad y sobrepeso. Nota descriptiva No. 311, enero del 2015 [Internet]. Disponible en: http://www.who.int/media centre/factsheets/fs311/es/.

2. Escobar ID, Rossi A. Sobrepeso y obesidad incrementan la epidemia de diabetes tipo 2. Diabetes al día. 2004. [Internet] Disponible en: http://encolombia.com/medicina/ revistas-medicas/d-diabetes/d22/sobrepeso-y-obesidad/

3. Resumen Ejecutivo ENSIN 2012. Encuesta Nacional de la situación nutricional en Colombia. [Internet] Disponible en: http://www.icbf.gov.co/portal/page/portal/Descargas 1/Resumenfi.pdf

4. Schaver PR, Kashyap SR, Wolski K et al. Bariatric Surgery versus Intensive Medical Therapy in Obese Patients with Diabetes. New England Journal of Medicine 2012;366:1567-157626.

5. Dixon JB, Zimmet P, Alberti KG, Rubino F. Bariatric surgery: an IDF statement for obese Type 2 diabetes. Diabetic Medicine $2011 ; 28: 628-642$. 
Experiencia del grupo de cirugía bariátrica y metabólica del Hospital Universitario de Neiva durante un año
R.F.S Revista Facultad de Salud Julio-Diciembre de 2015;7(2):15-22
6. Vélez JP, Arias R, Gómez S. Reintervenciones en cirugía bariátrica, conversión a otro procedimiento. Revista Colombiana de Cirugía. $2011 ; 26: 42-47$.

7. Ikramuddin S, Kellogg TA, Leslie DB. Laparoscopic conversión of vertical banded gastroplasty to a Roux-en-Y gastric bypass. Surgical Endoscopy. 2007;21:1927-30.

8. Parikh M, Pomp A, Gagner M. Laparoscopic conversion of failed gastric bypass to duodenal switch: Technical considerations and preliminary outcomes. Surgery for Obesity and Related Diseases. 2007;3:611-8.

9. McCormick JT, Papasavas PK, Caushaj PF et al. Laparoscopic revision of failed open bariatric procedures. Surgical Endoscopy. 2003;17:413-5.

10. Khaitan L, Van Sickle K, González R, et al. Laparoscopic revision of bariatric procedures: Is it feasible? The American Surgeon. 2005;71:6-10.

11. Marrodán MD, Montero V, Mesa M, et al. Percepción y atractivo de la imagen corporal: condicionantes biológicos y socioculturales. Zainak. 30, 2008, 15-28.

12. Riobó $P$, Kozarcewski M, Fernández JM. Obesidad en la mujer. Nutrición Hospitalaria. 2003;18:233-237.

13. Martin M, Beekley A, Kjorstad R, Sebesta J. Socioeconomic disparities in eligibility and access to bariatric surgery: a national population-based analysis. Surgery for Obesity and Related Diseases 6 (2010) 8-15.

14. Daes J, Restrepo H, Vélez JP, et al. Guías de manejo en cirugía. Cirugía Bariátrica. [Internet] Disponible en: http:/ /www.ascolcirugia.org/guiasCirugia/cirugiaBariatrica. pdf

15. Poirier $P$, Cornier $M$, et al. Bariatric Surgery and Cardiovascular Risk Factors A Scientific Statement From the American Heart Association. Circulation. 2011;123:1683-1701.

16. Leivonen MK, Juuti A, Jaser N, Mustonen H. Laparoscoxpic sleeve gastrectomy in patients over 59 years: early recovery and 12-month follow-up. Obesity Surgery. 2011 Aug;21(8):1180-7.

17. Khalaileh A, Matot I, Schweiger C, et al. Laparoscopic Roux-en-Y gastric bypass for the treatment of morbid obesity: experience with 50 patients. Israel Medical Association Journal 2008 May; 10(5):350-3.

18. Shabbir A, Loi TH, Lomanto D, et al. Surgical management of obesity - National University Hospital experience. Annals of the Academy of Medicine, Singapore. 2009 Oct;38(10):882-90.

19. Mingrone G, Panunzi S, De Gaetano A, et al. Bariatric Surgery versus Conventional Medical Therapy for Type 2 Diabetes. New England Journal of Medicine 2012;366:1577-1585.

20. Guh DP, Zhang W, Bansback N, et al. The incidence of comorbidities related to obesity and overweight: $A$ systematic review and meta-analysis. BMC Public Health 2009, 9:88.

21. Rubio MA, Martínez C, Vidal O, et al. Documento de consenso sobre cirugía bariátrica. Revista Española de Obesidad 2004;4:223-249.

22. Cabrerizo L, Rubio MA, Ballesteros MD, Moreno C. Complicaciones asociadas a la obesidad. Revista Española de Nutrición Comunitaria 2008;14(3):156-162.
23. Encinas A, Cañones PJ, Ortega RM, Requejo AM. Nutrición y enfermedades del aparato digestivo. Medicina General 2000;29:978-983.

24. López MJ, Soto A. Actualización en Obesidad. Cad Aten Primaria, Año 2010. Vol 17. Pág. 101-107.

25. Rodríguez LD, Vega M. Cirugía Bariátrica: Tratamiento de elección para la obesidad mórbida. Acta Médica Costarricense, vol 48 (4), Oct-Dic 2006.

26. Neligan P, Williams N. Non-surgical and surgical treatment of obesity. Anesthesiology Clinics of North America. 2005;23:501-523.

27. Kelum JM, DeMaria EJ, Sugerman HJ. The surgical treatment of obesity. American Journal of surgery. 1969;118:141-146.

28. Stocker D. Management of the bariatric surgery patient. Endocrinology and metabolism clinics of North America. 2003;32:437-457.

29. Livingston E. complication of bariatric surgery for obesity. The Surgical Clinics of North America 2005;85: 853-868.

30. Brolin RE, La Marca LB, Kenler HA, Cody RP. Malabsorptive Gastric Bypass in Patients With Superobesity. Journal of Gastrointestinal Surgery. 2002;6:195-205.

31. Brolin RE. Weight gain after short- and long-limb gastric bypass in patients followed for longer than 10 years. Annals of Surgery. 2007;246:163-4.

32. Stefanidis D, Kuwada TS, Gersin KS. The importance of the length of the limbs for gastric bypass patients an evidence-based review. Obesity Surgery. $2011 ; 211$ : 119-24.

33. Gurewithsch ED, Smith-Levitin M, Mack J. Pregnancy following gastric bypass surgery for morbid obesity. Obstet Gynecol 1998 Aug; 8 (4): 461-4.

34. Gagner M, Gumbs A. Gastric banding: Conversion to sleeve, bypass, or DS. Surgical Endoscopy. 2007;21:1931-5.

35. Gumbs AA, Pomp A, Gagner M. Revisional bariatric surgery for inadequate weight loss. Obesity Surgery. 2007; 17:1137-45.

36. Spann SJ, Nutting PA, Galliher JM, et al. Management of type 2 diabetes in the primary care setting: a practicebased research network study. Annals of Family Medicine 2006;4:23-31.

37. Buchwald H, Estok R, Fahrbach $K$, et al. Weight and type 2 diabetes after bariatric surgery: systematic review and meta-analysis. American Journal of Medicine 2009; 122:248-256.

38. Kashyap SR, Gatmaitan P, Brethaver S, Schaver P. Bariatric surgery for type 2 diabetes: Weighing the impact for obese patients. Cleveland Clinic Journal of Medicine. Vol. 77- 7. Julio 2010.

39. Balsiger B, Murr M, Poggio J, Sarr M. Bariatric surgery. The Medical Clinics of North America. 2000;84:477-489.

40. Cohen R. Cirugía gastrointestinal para los pacientes diabéticos tipo 2 no obesos. Revista Médica Clínica las Condes- 2009;20(5)703-707. 
R.F.S Revista Facultad de Salud

Julio-Diciembre de 2015;7(2):15-22
Experiencia del grupo de cirugía bariátrica y metabólica del Hospital Universitario de Neiva durante un año

41. Papapietro K, Díaz E, Csendes A, et al. Evolución de comorbilidades metabólicas asociadas a obesidad después de cirugía bariátrica. Revista Médica de Chile 2005;133:511-516.

42. Sjöström $L$, Lindroos $A$, Peltonen $M$, et al. Diabetes, and Cardiovascular Risk Factors 10 Years after Bariatric Surgery. New England Journal of Medicine 2004;351:2683-93.

43. Brethaver SA, Chand B, Schaver PR. Risks and benefits of bariatric surgery: Current evidence. Cleveland clinic journal of medicine Vol 73. 2006.
44. Ocón J, Pérez S, Gimeno S, Ruesca B, García R. Eficacia y complicaciones de la cirugía bariátrica en el tratamiento de la obesidad mórbida. Nutrición Hospitalaria. Vol. 20 No. 6 Madrid nov.-dic.

45. Keto JL, Kemmeter PR. Effect of Center of Excellence requirement by Centers for Medicare and Medicaid Services on practice trends. Surgery for Obesity and Related Diseases 4 (2008) 437-440. 